\title{
Toward quantum chemical calculations of open shell molecules on quantum computers
}

Kenji Sugisaki, ${ }^{1,2}$ Shigeaki Nakazawa, ${ }^{1}$ Kazuo Toyota, ${ }^{1}$ Kazunobu Sato, ${ }^{1}$ Daisuke Shiomi, ${ }^{1}$ and Takeji Takui ${ }^{1,3}$

${ }^{1}$ Graduate School of Science, Osaka City University

2 JST PRESTO

${ }^{3}$ Support Department/University Research Administrator Center, Osaka City University
O S A K A
C I $\mathbf{T} Y$

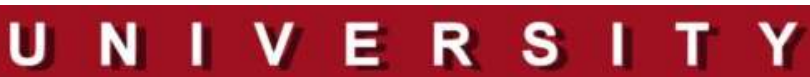




\section{Table of Contents}

$\square$ Introduction

$\checkmark$ Quantum chemical calculations on quantum computers

$\checkmark$ Open shell molecules

$\checkmark$ Construction of spin symmetry-adapted wave functions

K. Sugisaki et al, J. Phys. Chem. A 2016, 120, 6459-6466.

K. Sugisaki et al, Chem. Phys. Lett.: X 2019, 1, 100002.

K. Sugisaki et al, ACS Cent. Sci. 2019, 5, 167-175.

$\square$ Determination of Spin Quantum Number $S$

$\checkmark$ Time evolution of wave function under the spin operator $\mathbf{S}^{2}$

$\checkmark$ Trotter decomposition error analysis

$\checkmark$ Spin state discrimination by quantum phase estimation

K. Sugisaki et al, Phys. Chem. Chem. Phys. 2019, 21, 15356-15361.

$\square$ Summary 


\section{Quantum Computers}

$$
H|\Psi\rangle=E|\Psi\rangle
$$

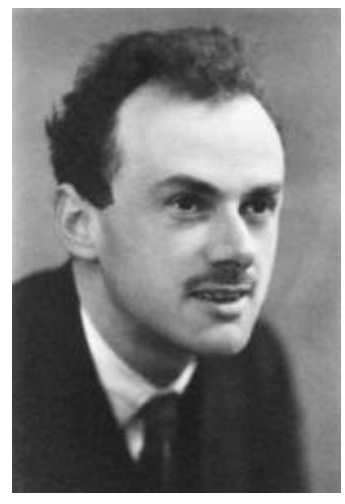

The underlying physical lows necessary for the mathematical theory of a large part of physics and the whole of chemistry are thus completely known, and the difficulty is only that the exact application of these laws leads to equations much too complicated to be soluble. It therefore becomes desirable that approximate practical methods of applying quantum mechanics should be developed, ...

P. A. M. Dirac, Proc. R. Soc. London Ser. A 1929, 123, 714.

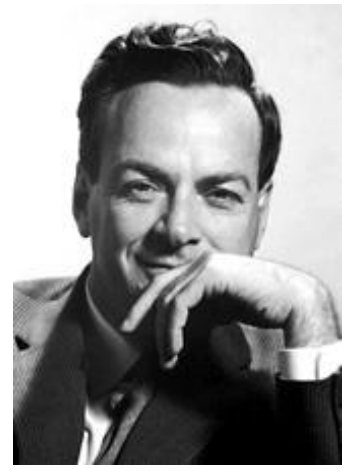

How can we simulate the quantum mechanics? Let the computer itself be built of quantum mechanical elements which obey quantum mechanical laws.

R. P. Feynman, Int. J. Theor. Phys. 1982, 21, 467. 


\section{Quantum Chemistry on Quantum Computers ${ }^{[1]}$}

\section{Quantum Phase Estimation ${ }^{[2]}$}

- Simulate time evolution of wave function on quantum computers

- Obtain full-Cl energy by inverse quantum Fourier transformation

- Deep quantum circuit: Difficult to execute on NISQ devices

\section{Variational quantum eigensolver (VQE) ${ }^{[3]}$}

- Quantum-classical hybrid computation model

- Use parametrized quantum circuits to generate approximate wave function, and use classical devices to optimize parameters to minimize energy expectation values

[1] Y. Cao et al, Chem. Rev. 2019, 119, 10856-10915., and references therein.

[2] A. Aspuru-Guzik et al, Science 2005, 39, 1704-1707.

[3] M.-H. Yung et al, Sci. Rep. 2014, 4, 3589; A. Peruzzo et al, Nat. Comm. 2014, 5, 4213. 


\section{Quantum Phase Estimation (QPE) Algorithm ${ }^{[4]}$}

$$
\exp (-i H t)|\Psi\rangle=\exp (-i E t)|\Psi\rangle=\exp (-i 2 \pi \phi)|\Psi\rangle
$$

Time evolution of wave function

Phase shift depending on energy

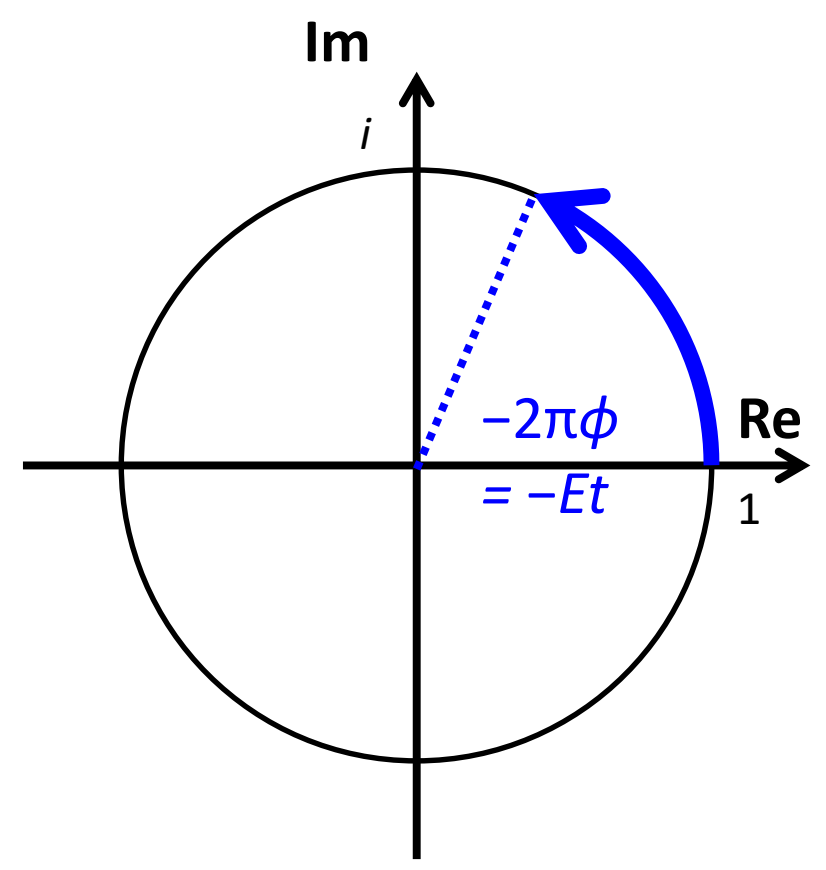

$$
E t=2 \pi \phi
$$

Inverse Fourier transformation

(Time domain $\rightarrow$ Frequency domain)

[4] A. Abrams, S. Lloyd, Phys. Rev. Lett. 1999, 83, 5162-5165. 


\section{N-Qubit QPE}

$\exp (-i H t)|\Psi\rangle=\exp (-i E t)|\Psi\rangle=\exp (-i 2 \pi \phi)|\Psi\rangle$

$H_{d}|0\rangle=\frac{1}{\sqrt{2}}(|0\rangle+|1\rangle)$

$\phi=0 . x_{1} x_{2} x_{3} \cdots x_{j}$

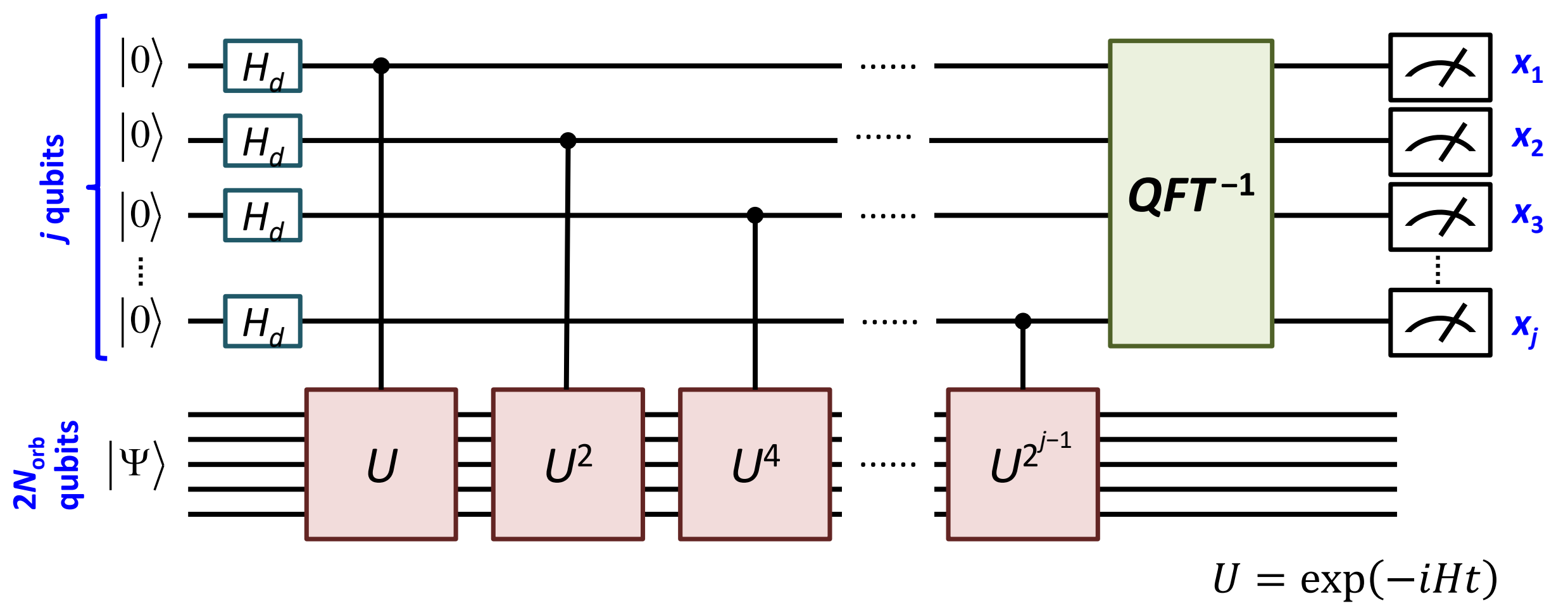




\section{The QPE-Based Full-CI Method}

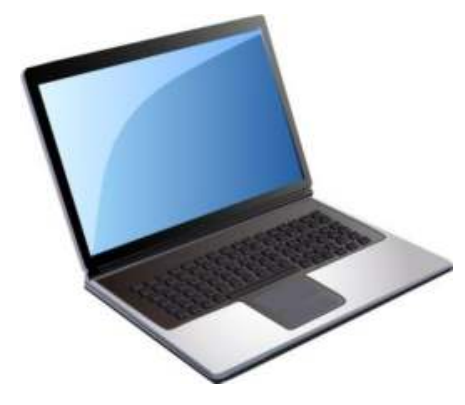

Using CLASSICAL computers

$\checkmark$ Preparation of initial guess wave functions

Perform Hartree-Fock calculations

$\checkmark$ Compute one- and two-electron integrals

$\checkmark$ Transform molecular Hamiltonian to qubit operators

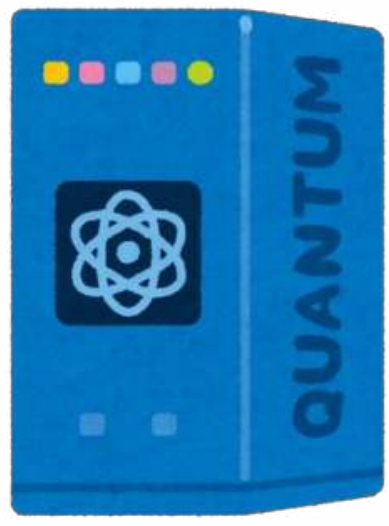

\section{Using QUANTUM computers}

$\checkmark$ Map the wave function onto quantum registers

$\checkmark$ Simulate the time evolution of wave functions

$\checkmark$ Execute inverse QFT and readout Full-Cl energy 


\section{Assumptions in QPE-Based Algorithms}

- Hartree-Fock wave function is available.

Sometimes Hartree-Fock calculations do not converge in large molecules like proteins. Application of QCC-on-QCs for such systems is still an open question.

- Hartree-Fock wave function is a good approximation of the ground state wave function.

This assumption is not always correct. In open shell molecules containing unpaired electrons of spin- $\beta$. Use symmetry adapted wave function is crucial.

K. Sugisaki et al, J. Phys. Chem. A 2016, 120, 6459-6466.

K. Sugisaki et al, Chem. Phys. Lett.: X 2019, 1, 100002.

K. Sugisaki et al, ACS Cent. Sci. 2019, 5, 167-175. 


\section{Covalent Bond Dissociations}
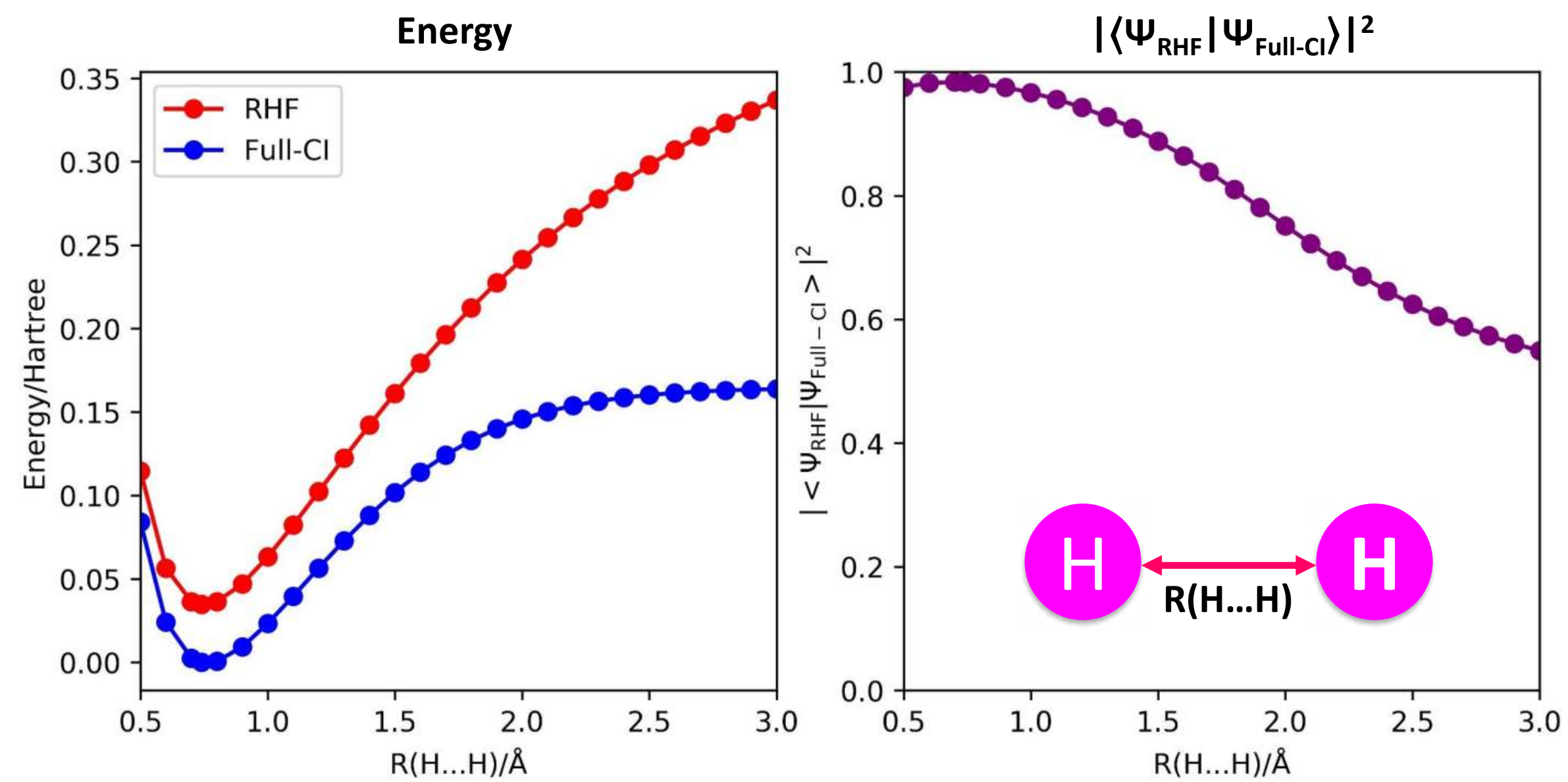

RHF method is not able to describe bond dissociation limit correctly.

Molecules undergoing bond dissociation are representatives of open shell systems. 


\section{Open Shell Systems}

Not just reactive intermediates and unusual molecules, but fundamental chemical species!

Spin qubit molecules

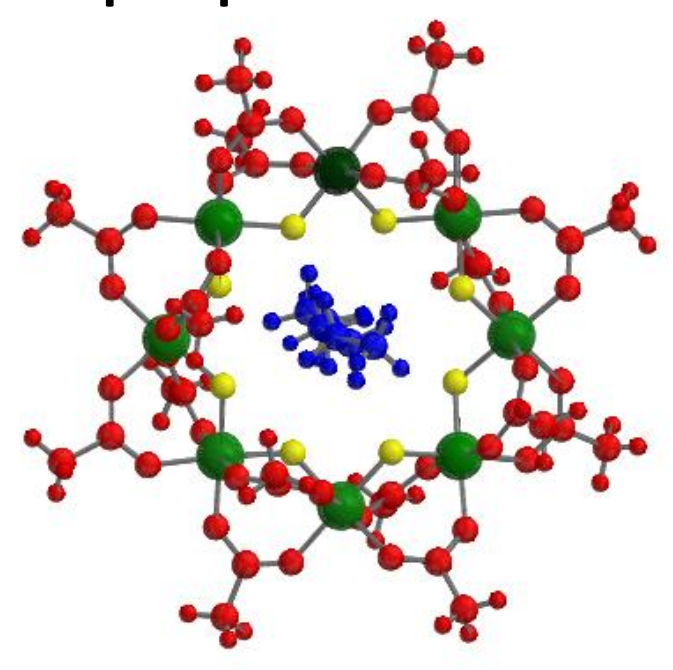

Nanographenes

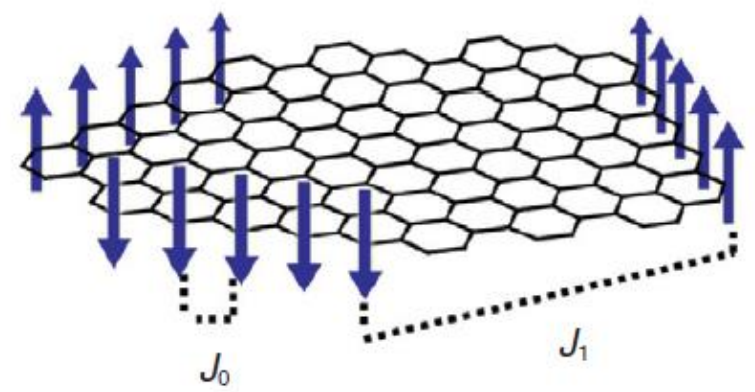

Molecules under covalent bond dissociations

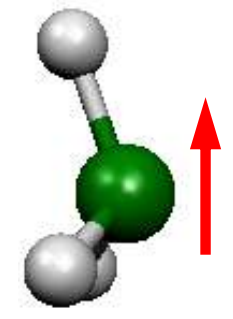

Stacked $\pi$ radicals

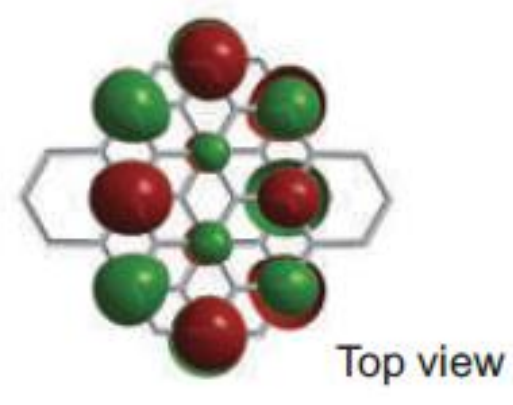

Mn cluster in PS-II
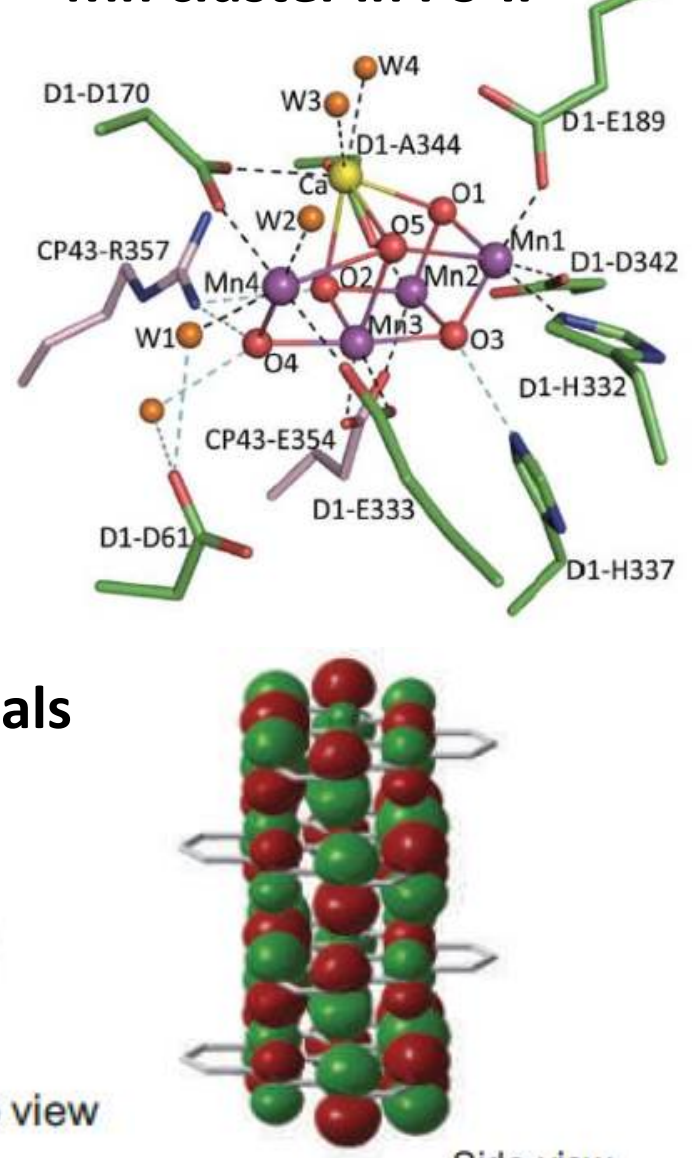


\section{Use Symmetry-Adapted Wave Functions!}

\section{Quantum Chemistry on Quantum Computers: A Polynomial-Time Quantum Algorithm for Constructing the Wave Functions of Open- Shell Molecules}

Kenji Sugisaki, * Satoru Yamamoto, Shigeaki Nakazawa, Kazuo Toyota, Kazunobu Sato, * Daisuke Shiomi, and Takeji Takui*

Department of Chemistry and Molecular Materials Science, Graduate School of Science, Osaka City University, 3-3-138 Sugimoto, Sumiyoshi-ku, Osaka 558-8585, Japan

Supporting Information

ABSTRACT: Quantum computers are capable to efficiently perform full configuration interaction (FCI) calculations of atoms and molecules by using the quantum phase estimation (QPE) algorithm. Because the success probability of the QPE depends on the overlap between approximate and exact wave functions, efficient methods to prepare accurate initial guess wave functions enough to have sufficiently large overlap with the exact ones are highly desired. Here, we propose a quantum algorithm to construct the wave function consisting of one configuration state function, which is suitable for the initial

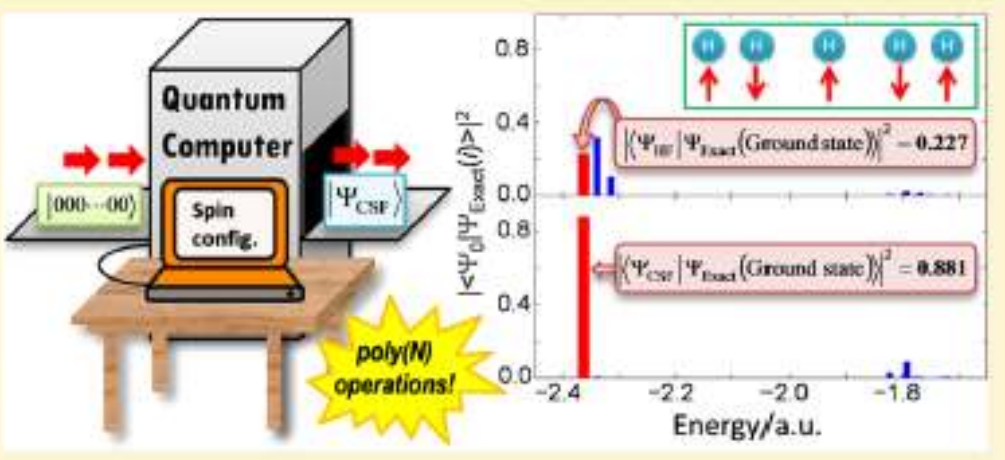
guess wave function in QPE-based FCI calculations of openshell molecules, based on the addition theorem of angular momentum. The proposed quantum algorithm enables us to prepare the wave function consisting of an exponential number of Slater determinants only by a polynomial number of quantum operations. 


\section{Use Symmetry-Adapted Wave Functions!}
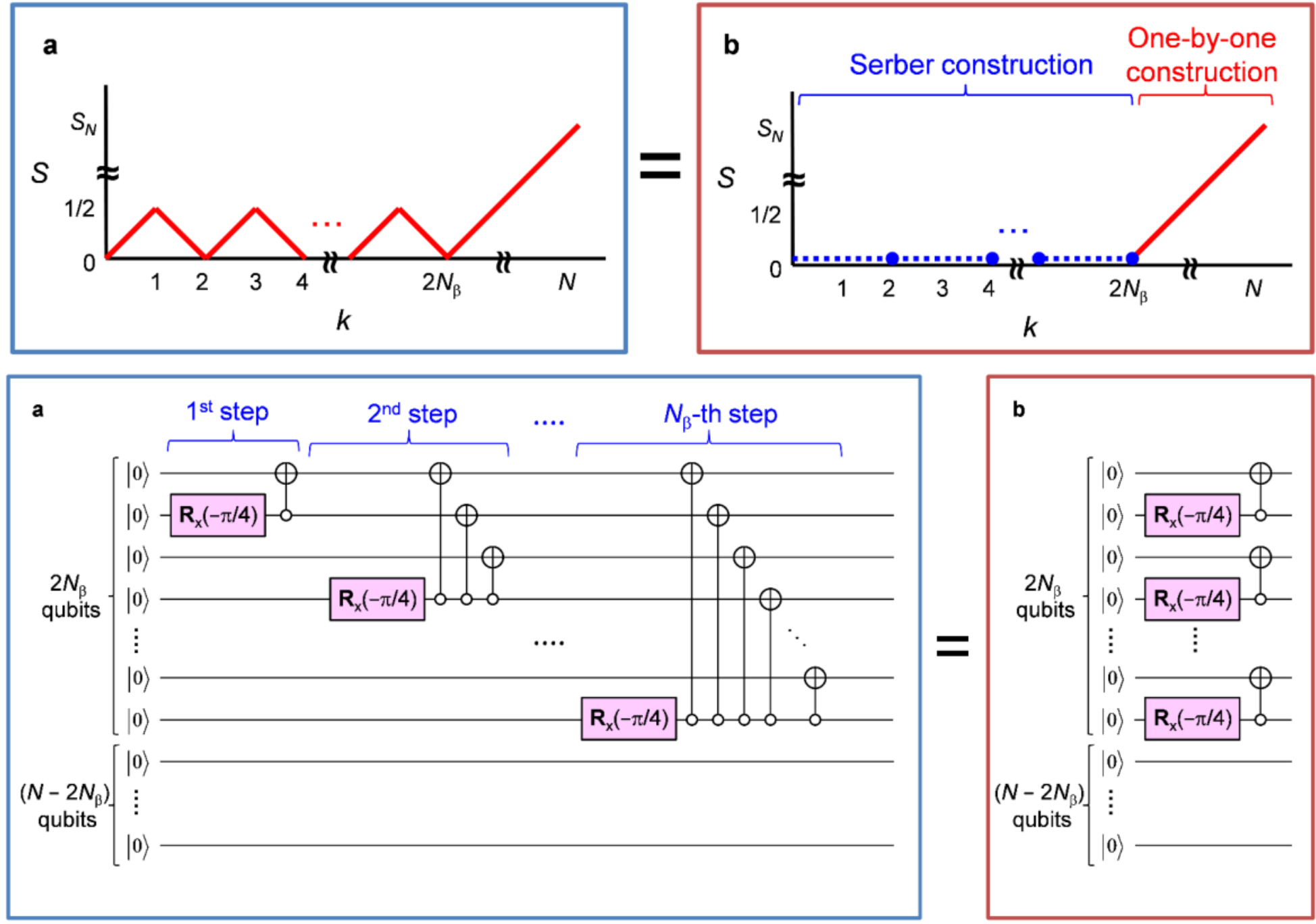

K. Sugisaki et al, J. Phys. Chem. A 2016, 120, 6459-6466.

K. Sugisaki et al, Chem. Phys. Lett.: X 2019, 1, 100002. 


\title{
Use Multi-Configurational Wave Functions!
}

\section{central science}

\begin{tabular}{lr} 
Research Article \\
\hline C Cite This: ACS Cent 5d. 2019, 5, 167-175
\end{tabular}

Quantum Chemistry on Quantum Computers: A Method for Preparation of Multiconfigurational Wave Functions on Quantum Computers without Performing Post-Hartree-Fock Calculations

Kenji Sugisaki,* ${ }^{\dagger} \odot$ Shigeaki Nakazawa, ${ }^{\dagger}$ Kazuo Toyota, ${ }^{\dagger}$ Kazunobu Sato,* ${ }^{*} \odot$ Daisuke Shiomi, and Takeji Takui ${ }^{*}+t_{0}$

${ }^{\dagger}$ Department of Chemistry and Molecular Materials Science, Graduate School of Science, Osaka City University, 3-3-138 Sugimoto, Sumiyoshi-ku, Osaka 558-8585, Japan

${ }^{\text {† }}$ Research Support Department/University Research Administrator Center, University Administration Division, Osaka City University, 3-3-138 Sugimoto, Sumiyoshi-ku, Osaka 558-8585, Japan

(3) Supporting Information

\begin{abstract}
The full configuration interaction (full-Cl) method is capable of providing the numerically best wave functions and energies of atoms and molecules within basis sets being used, although it is intractable for classical computers. Quantum computers can perform full-CI calculations in polynomial time against the system size by adopting a quantum phase estimation algorithm (QPEA). In the QPEA the preparation of initial guess wave functions having sufficiently large overlap with the exact wave function is recommended. The Hartree-Fock (HF) wave function is a good initial guess only for closed shell singlet molecules and high-spin molecules carrying no spin- $\beta$ unpaired electrons, around their equilibrium geometry, and thus, the construction

of multiconfigurational wave functions without performing post-HF calculations on classical computers is highly desired for applying the method to a wide variety of chemistries and physics. In this work, we propose a method to construct multiconfigurational initial guess wave functions suitable for QPEA-based full-CI calculations on quantum computers, by utilizing diradical characters computed from spin-projected UHF wave functions. The proposed approach drastically improses the wave function overlap, particularly in molecules with intermediate diradical characters.
\end{abstract}

For more detail, please see my poster!

https://www.morressier.com/article/multiconfigurational-quantum-chemistry-quantumcomputers/5e73d6ce139645f83c22b0ec 


\section{Importance of Spin States in Chemistry}

Spin-triplet oxygen

$\mathrm{O}_{2}$

Necessary for life

Spin-singlet oxygen

One of the reactive oxygen species (ROS) Can be the cause of various diseases

Different spin states exhibit different properties

How to determine the quantum states obtained from quantum simulations? 


\section{Determination of Spin Quantum Number S}

How to determine the spin quantum number of arbitrary wave functions?

$>$ Check whether the quantum simulation converged to desired state or not

$>$ Calculate ground state first, then determine its spin quantum number

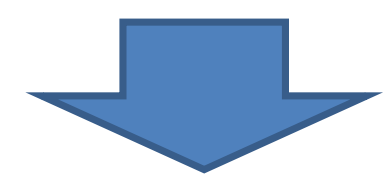

Simulating the time evolution of wave function using the spin operator $\mathbf{S}^{2}$ instead of Hamiltonian!!

$$
\exp \left(-i \mathbf{S}^{2} t\right)|\Psi\rangle=\exp \{-i S(S+1) t\}|\Psi\rangle
$$




\section{Quantum Simulations with $S^{2}$ Operator}

Simulate time evolution of wave function $\exp \left(-i S^{2} t\right)|\Psi\rangle$ on quantum computers

$$
\exp \left(-i \mathbf{S}^{2} t\right)|\Psi\rangle=\exp \{-i S(S+1) t\}|\Psi\rangle
$$

Second quantization $\rightarrow$ Pauli operators $\rightarrow$ Quantum circuits

$\mathbf{S}^{2}$ operator can be defined as follows ${ }^{[5]}$ :

$$
\begin{aligned}
& \mathbf{S}^{2}=\sum_{p, q}^{N} \mathbf{S}(p) \cdot \mathbf{S}(q)=\sum_{p, q}^{N}\left[\mathbf{S}_{Z}(p) \mathbf{S}_{Z}(q)+\frac{1}{2}\left\{\mathbf{S}_{+}(p) \mathbf{S}_{-}(q)+\mathbf{S}_{-}(p) \mathbf{S}_{+}(q)\right\}\right] \\
& \mathbf{S}_{Z}(p) \mathbf{S}_{Z}(q)=\frac{1}{4}\left(a_{p \alpha}^{\dagger} a_{p \alpha} a_{q \alpha}^{\dagger} a_{q \alpha}+a_{p \beta}^{\dagger} a_{p \beta} a_{q \beta}^{\dagger} a_{q \beta}-a_{p \alpha}^{\dagger} a_{p \alpha} a_{q \beta}^{\dagger} a_{q \beta}-a_{p \beta}^{\dagger} a_{p \beta} a_{q \alpha}^{\dagger} a_{q \alpha}\right) \\
& \mathbf{S}_{+}(p) \mathbf{S}_{-}(q)+\mathbf{S}_{-}(p) \mathbf{S}_{+}(q)=a_{p \alpha}^{\dagger} a_{p \beta} a_{q \beta}^{\dagger} a_{q \alpha}+a_{p \beta}^{\dagger} a_{p \alpha} a_{q \alpha}^{\dagger} a_{q \beta}
\end{aligned}
$$

[5] R. Pauncz, The Construction of spin eigenfunctions. An Exercise Book, Kluwer/Plenum, 2000. 


\section{Jordan-Wigner Transformation}

Second quantized Hamiltonian $\rightarrow$ Qubit Hamiltonian

(Creation/annihilation operators $\rightarrow$ Strings of Pauli operators)

$$
\mathrm{X}_{p}=\sigma_{p}{ }^{\mathrm{X}}, \mathrm{Y}_{p}=\sigma_{p}{ }^{\mathrm{Y}}, \mathrm{Z}_{p}=\sigma_{p}{ }^{\mathrm{Z}}
$$

$$
a_{p}^{\dagger}=\frac{1}{2}\left(X_{p}-i Y_{p}\right)\left(\prod_{q>p} Z_{q}\right) \quad a_{p}=\frac{1}{2}\left(X_{p}+i Y_{p}\right)\left(\prod_{q>p} Z_{q}\right)
$$

$S^{2}$ operator: two molecular orbital systems

$$
\begin{gathered}
\mathbf{S}^{2}=0.75-0.375\left(Z_{0} Z_{1}+Z_{2} Z_{3}\right)+0.125\left(Z_{0} Z_{2}+Z_{1} Z_{3}-Z_{0} Z_{3}-Z_{1} Z_{2}\right) \\
+0.125\left(X_{1} X_{2} X_{3} X_{4}+X_{1} X_{2} Y_{3} Y_{4}+X_{1} Y_{2} X_{3} Y_{4}+Y_{1} X_{2} Y_{3} X_{4}\right. \\
\left.+Y_{1} Y_{2} X_{3} X_{4}+Y_{1} Y_{2} Y_{3} Y_{4}-X_{1} Y_{2} Y_{3} X_{4}-Y_{1} X_{2} X_{3} Y_{4}\right)
\end{gathered}
$$




\section{Quantum Circuits for $\exp \left(-i S^{2} t\right)|\Psi\rangle$}

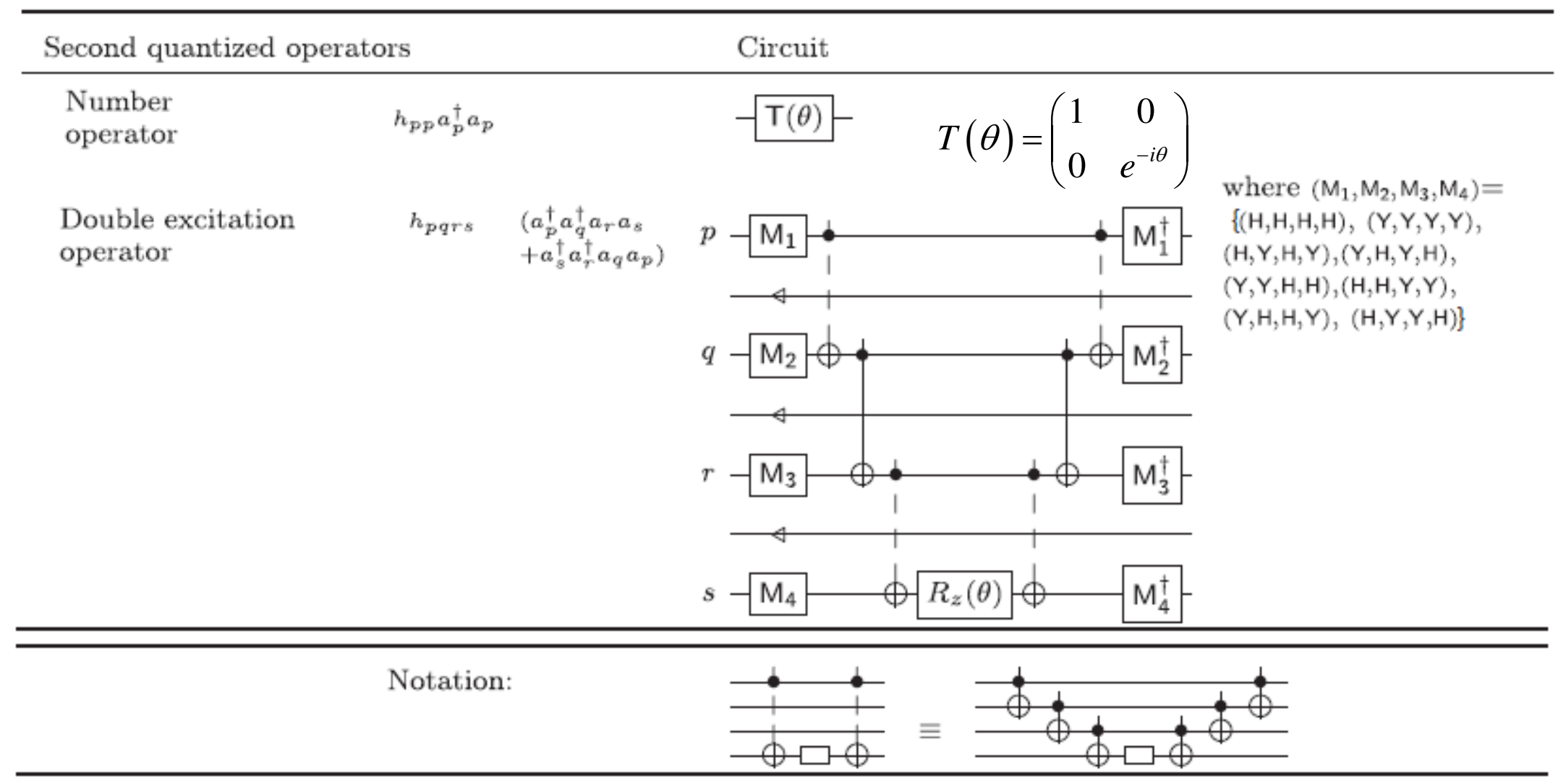

$\mathrm{H}_{2}$ in STO-3G basis (2 orbitals) 76 single-qubit gates and 54 CNOT gates (Jordan-Wigner transformation) 32 single-qubit gates and 46 CNOT gates (Bravyi-Kitaev transformation)

J. D. Whitfield, J. Biamonte, A. Aspuru-Guzik, Mol. Phys. 2011, 109, 735-750. 


\section{Generalized Spin Coordinate Mapping (GSCM)}

\begin{tabular}{|c|c|c|}
\hline & JWT & GSCM \\
\hline Unoccupied & $|00\rangle$ & $|00\rangle$ \\
\hline Occupied by spin- $\beta$ & $|01\rangle$ & $|11\rangle$ \\
\hline Occupied by spin- $\alpha$ & $|10\rangle$ & $|10\rangle$ \\
\hline Doubly occupied & $|11\rangle$ & $|01\rangle$ \\
\hline
\end{tabular}

1st qubit: Open shell $(|1\rangle)$ or not $(|0\rangle)$

2nd qubit: Occupied by spin- $\beta$ electron $(|1\rangle)$ or not (|0|)

CNOT operations

GSCM 


\section{Quantum Simulations with $S^{2}$ Operator}

$\mathbf{S}^{2}$ operator can also be defined as follows ${ }^{[5]}$ :

$$
\begin{aligned}
& \mathbf{S}^{2}=\sum_{p, q}^{N} \mathbf{S}(p) \cdot \mathbf{S}(q)=\sum_{p}^{N} \frac{3}{4} n_{p}^{\text {spin }}+\sum_{p \neq q}^{N}\left(-\frac{1}{4} n_{p}^{\text {spin }} n_{q}^{\text {spin }}+\frac{1}{2} \mathbf{P}_{p q}\right) \\
& n_{p}{ }^{\text {spin: }} \text { Number operator of spin acting on the } p \text {-th molecular orbital } \\
& \mathbf{P}_{p q}\left|\cdots \alpha_{p} \cdots \alpha_{q} \cdots\right\rangle \longrightarrow\left|\cdots \alpha_{p} \cdots \alpha_{q} \cdots\right\rangle
\end{aligned}
$$

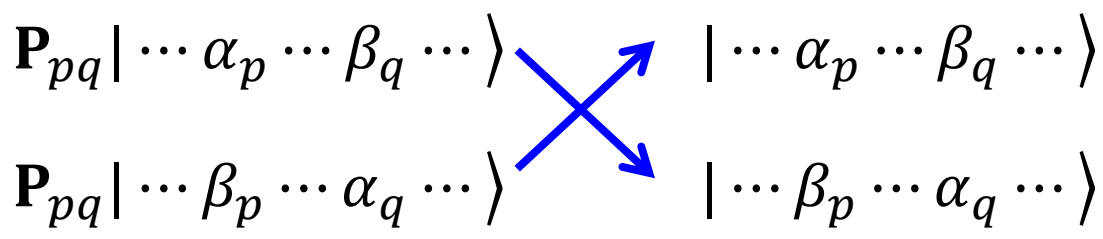

$$
\begin{aligned}
& \mathbf{P}_{p q}\left|\cdots \beta_{p} \cdots \beta_{q} \cdots\right\rangle \longrightarrow\left|\cdots \beta_{p} \cdots \beta_{q} \cdots\right\rangle \\
& {\left[n_{p}^{\text {spin }}, n_{q}^{\text {spin }}\right]=0} \\
& {\left[n_{p}^{\text {spin }}, \mathbf{P}_{p q}\right]=0} \\
& {\left[\mathbf{P}_{p q}, \mathbf{P}_{p r}\right] \neq 0}
\end{aligned}
$$

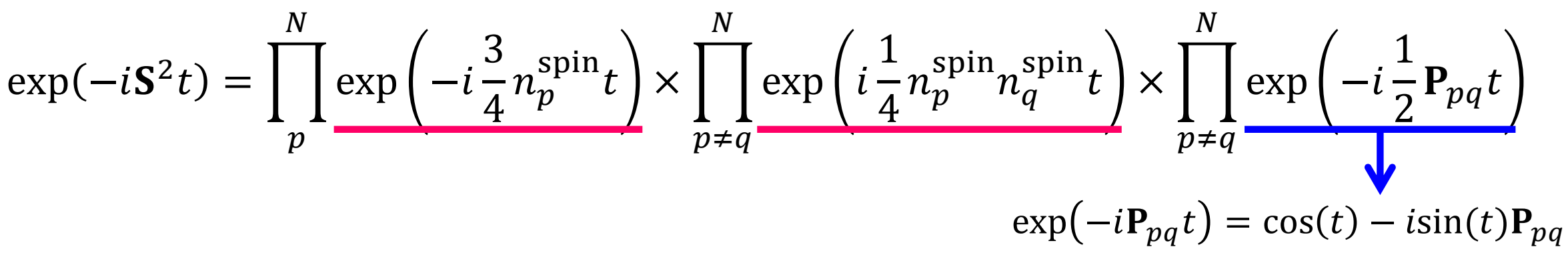

[5] R. Pauncz, The Construction of spin eigenfunctions. An Exercise Book, Kluwer/Plenum, 2000. 


\section{Quantum Circuit}

$\exp \left(-i \mathbf{S}^{2} t\right)=\prod_{p}^{N} \exp \left(-i \frac{3}{4} n_{p}^{\text {spin }} t\right) \times \prod_{p \neq q}^{N} \exp \left(i \frac{1}{4} n_{p}^{\text {spin }} n_{q}^{\text {spin }} t\right) \times \prod_{p \neq q}^{N} \exp \left(-i \frac{1}{2} \mathbf{P}_{p q} t\right)$

(1)

(2)

(3)

(4)

(5) $\theta=t / \pi$

$\left|\varphi_{1 \alpha}\right\rangle$

$\left|\varphi_{1 \beta}\right\rangle$

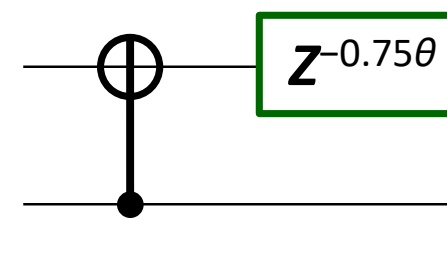

$\left|\varphi_{2 \alpha}\right\rangle$

$\left|\varphi_{2 \beta}\right\rangle$

$|0\rangle$

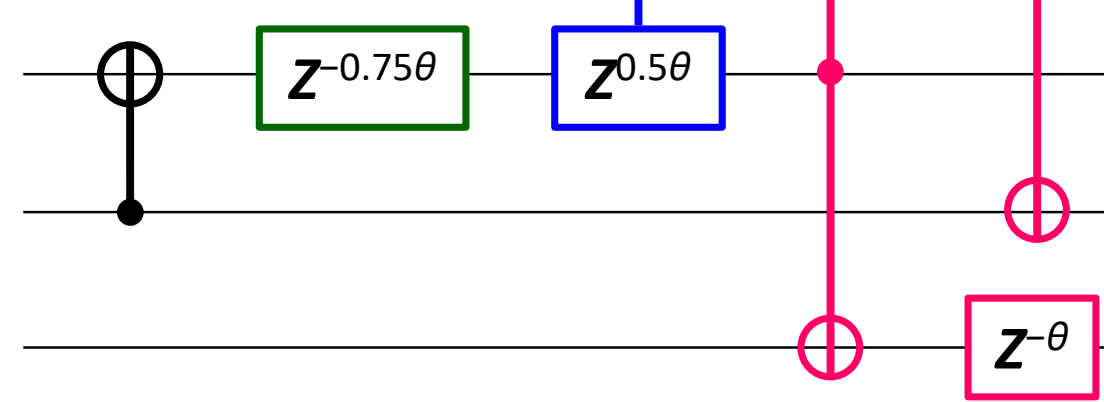

(1) Transform DM to GSCM

(2) The first term

(3) The second term

(4) The third term

(5) Back transformation from GSCM to DM

Trotter decomposition is required only for $P_{p q}$ terms! 


\section{Numerical Simulations of $\exp \left(-i S^{2} t\right)|\psi\rangle$}

$\exp \left(-i \mathbf{S}^{2} t\right)=\prod_{p}^{N} \exp \left(-i \frac{3}{4} n_{p}^{\text {spin }} t\right) \times \prod_{p \neq q}^{N} \exp \left(i \frac{1}{4} n_{p}^{\text {spin }} n_{q}^{\text {spin }} t\right) \times \prod_{p \neq q}^{N} \exp \left(-i \frac{1}{2} \mathbf{P}_{p q} t\right)$

- Python program using OpenFermion ${ }^{[6]}$ and Cirq $^{[7]}$ libraries

- $t=2 \pi$ (Total time of evolution)

-1st order Trotter decomposition

Initial wave functions ... $S^{2}$ eigenfunctions

$$
\begin{aligned}
& |\Psi(S=0)\rangle=\frac{1}{\sqrt{2}}(|\alpha \beta\rangle-|\beta \alpha\rangle) \\
& |\Psi(S=1)\rangle=\frac{1}{\sqrt{2}}(|\alpha \beta\rangle+|\beta \alpha\rangle) \\
& |\Psi(S=1 / 2)\rangle=\frac{1}{\sqrt{6}}(2|\alpha \alpha \beta\rangle-|\alpha \beta \alpha\rangle-|\beta \alpha \alpha\rangle)
\end{aligned}
$$




\section{Numerical Simulations of $\exp \left(-i S^{2} t\right)|\psi\rangle$}

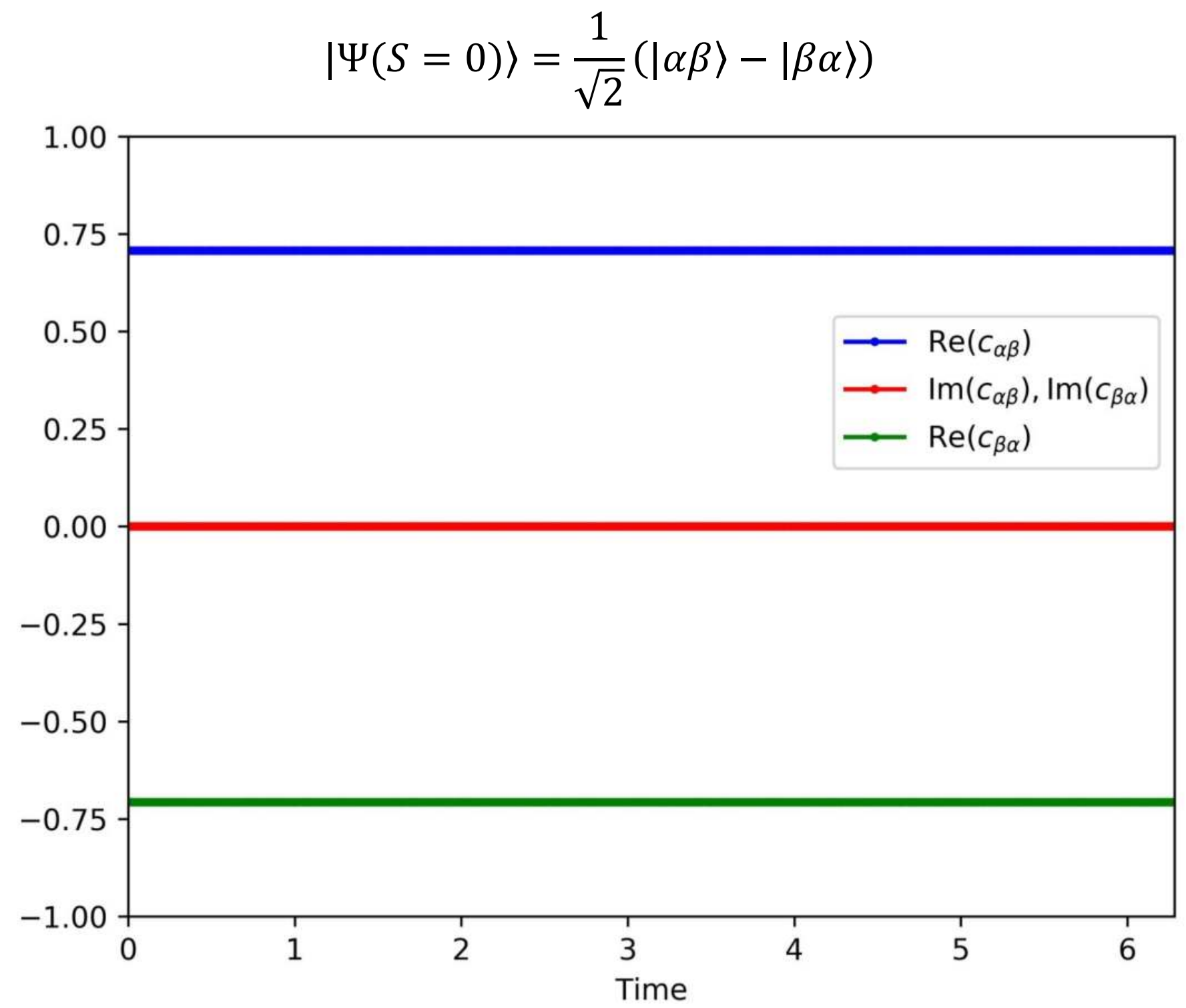




\section{Numerical Simulations of $\exp \left(-i S^{2} t\right)|\psi\rangle$}

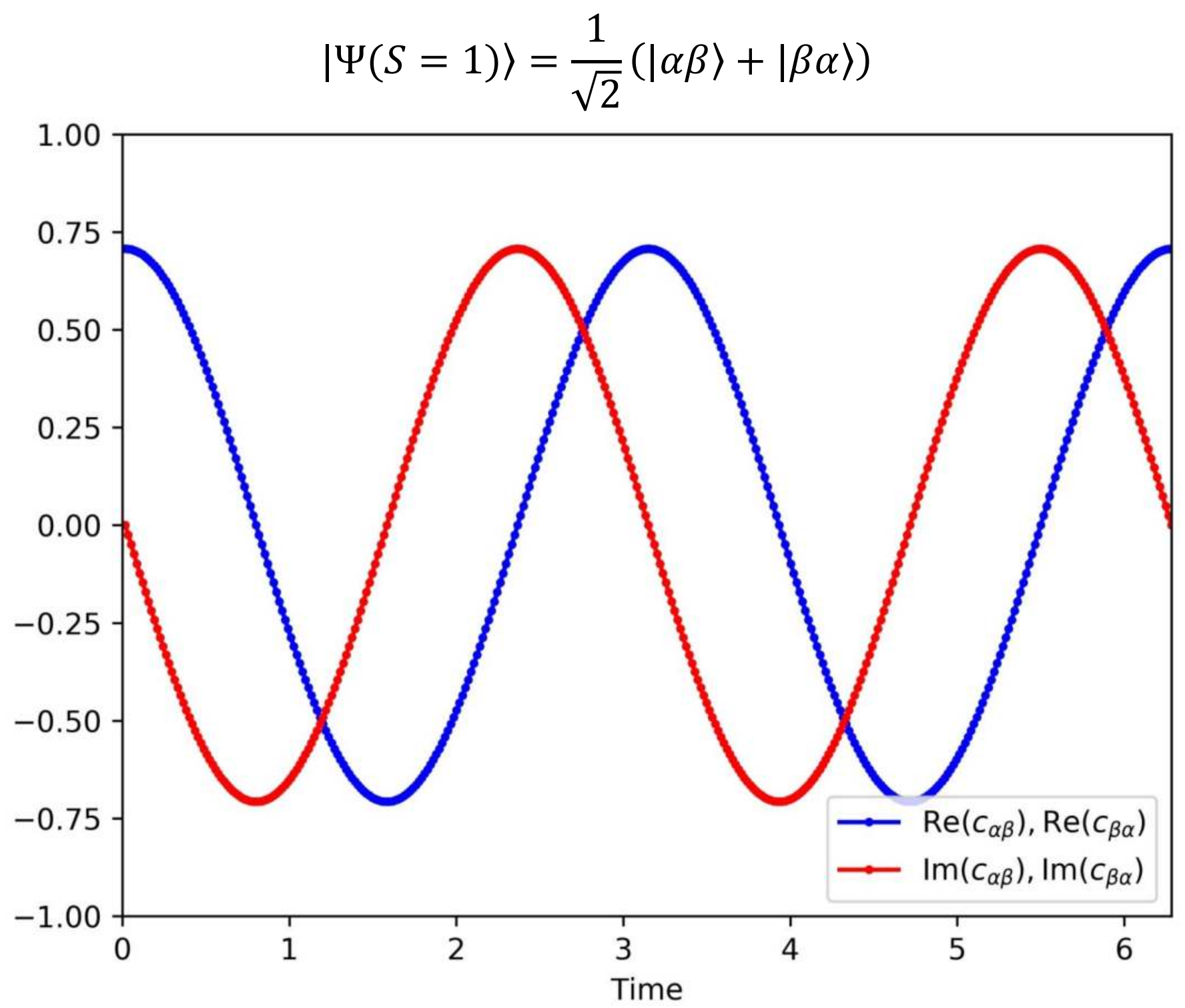




\section{Numerical Simulations of $\exp \left(-i S^{2} t\right)|\psi\rangle$}

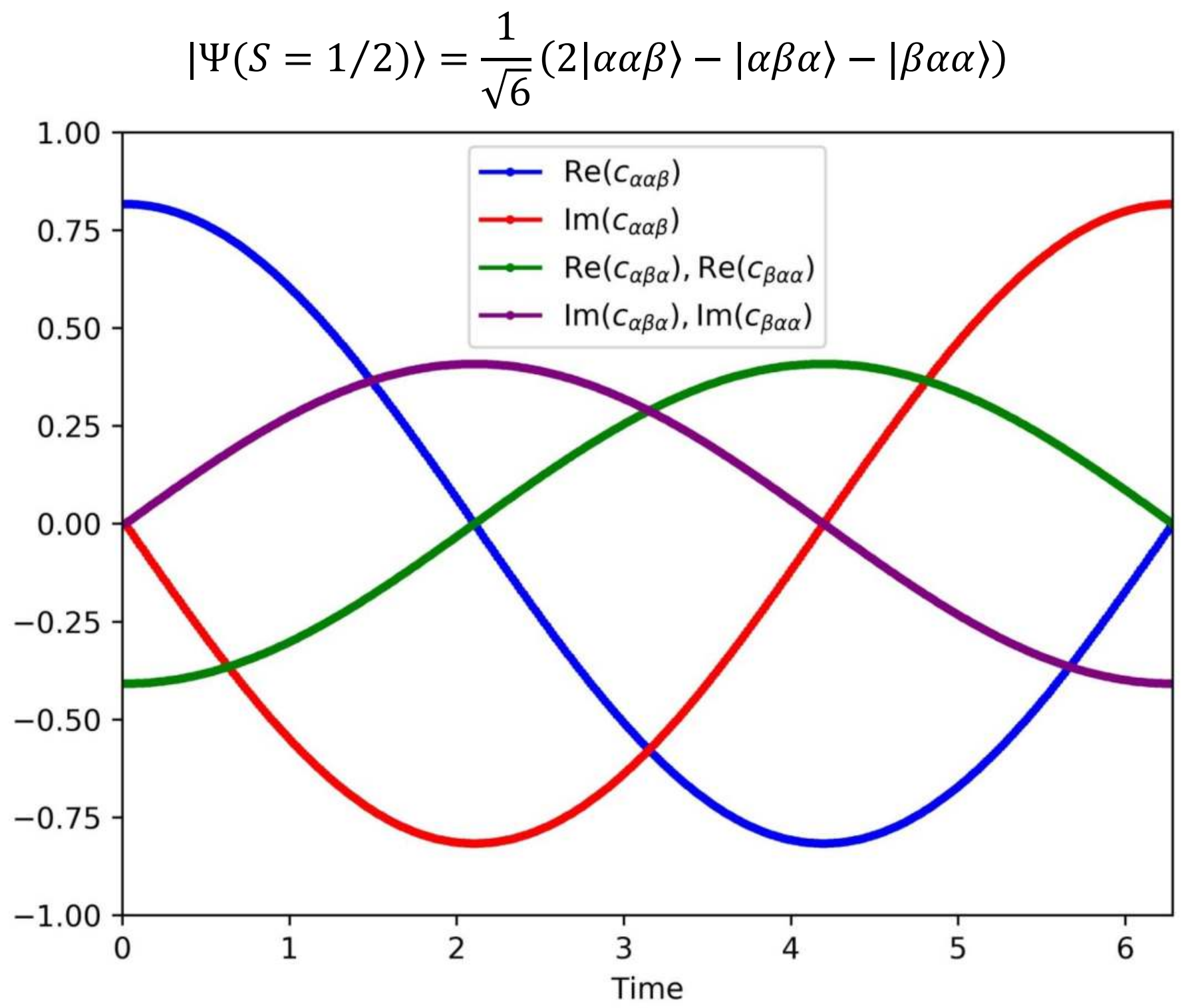




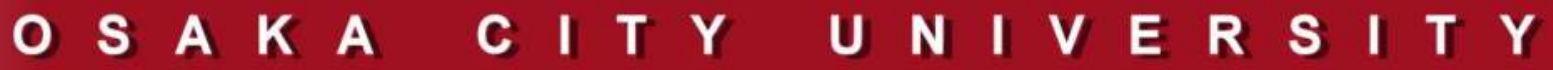

\section{Trotter Decomposition Error Analysis}

$$
e^{-i(A+B+\cdots+Z) t} \approx\left(e^{-i A t / N} e^{-i B t / N} \times \cdots \times e^{-i Z t / N}\right)^{N}
$$

Simulation conditions: The system consists of 6 electrons in 12 spin orbitals Randomly generated initial states with $M_{S}=0(S \neq 0)$ Evolution time $t=10$

First order Trotter decomposition, Lexicographic order

\section{GSCM}

JWT $\quad\left|\left\langle\Psi_{\text {Exact }} \mid \Psi_{\text {Sim }}\right\rangle\right|^{2}$
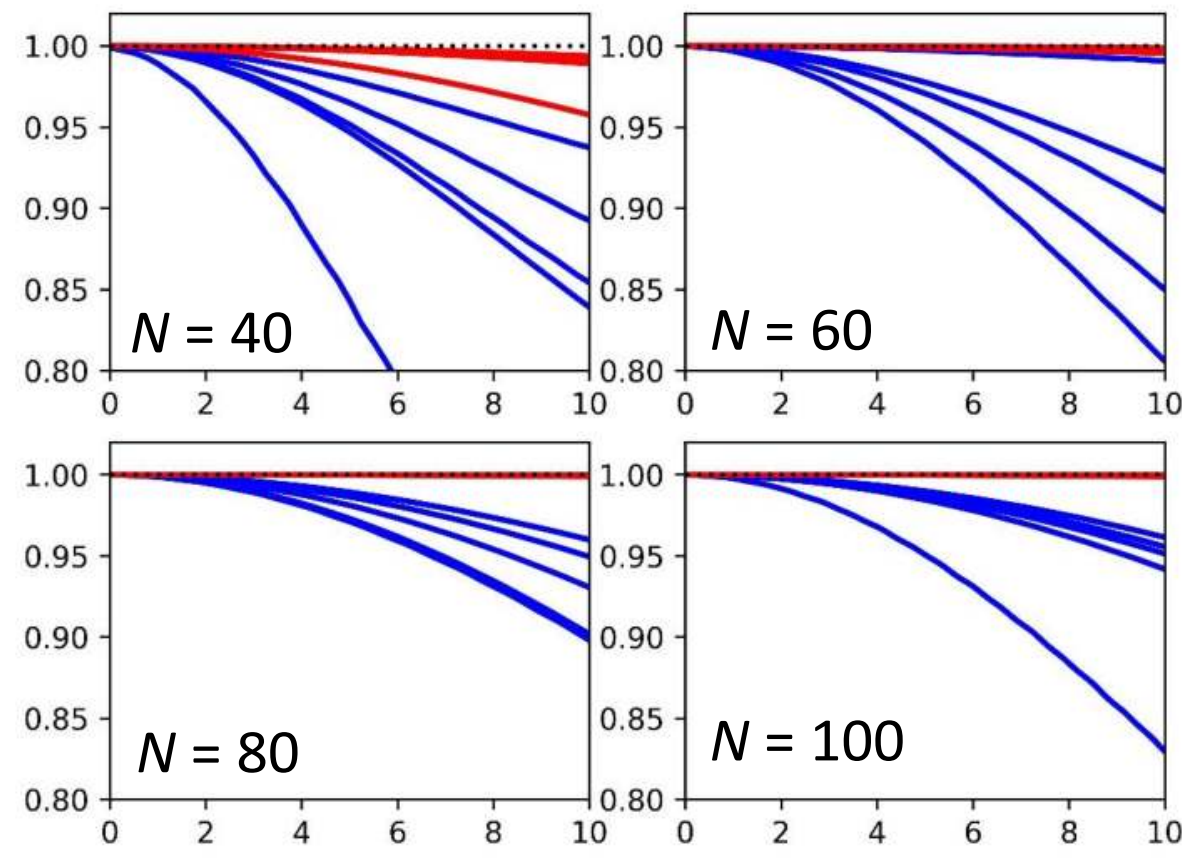

Time $t$ $\left\langle S^{2}\right\rangle_{\text {Sim }}-\left\langle S^{2}\right\rangle_{\text {Exact }}$
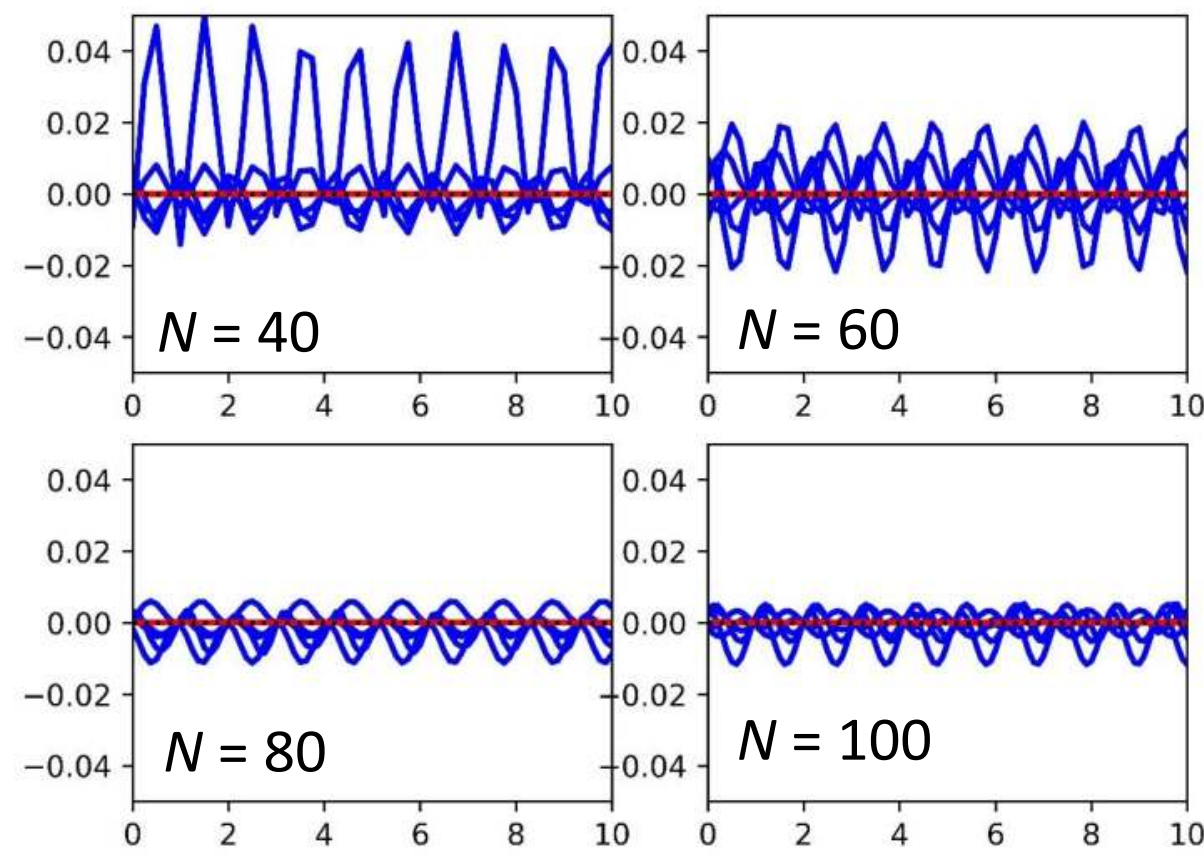

Time $t$ 


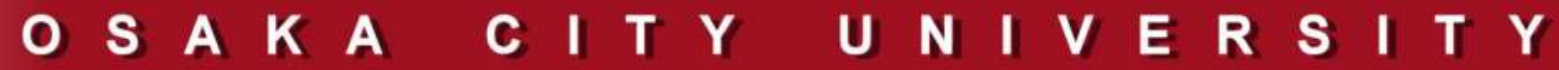

\section{Trotter Decomposition Error Analysis: Second-Order Trotter Decomposition}

Second order Trotter decomposition

$$
e^{-i(A+B+\cdots+Z) t} \approx\left(e^{-i A t / 2 N} e^{-i B t / 2 N} \times \cdots \times e^{-i Z t / N} \times \cdots \times e^{-i B t / 2 N} e^{-i A t / 2 N}\right)^{N}
$$

\section{GSCM}

JWT $\quad\left|\left\langle\Psi_{\text {Exact }} \mid \Psi_{\text {Sim }}\right\rangle\right|^{2}$
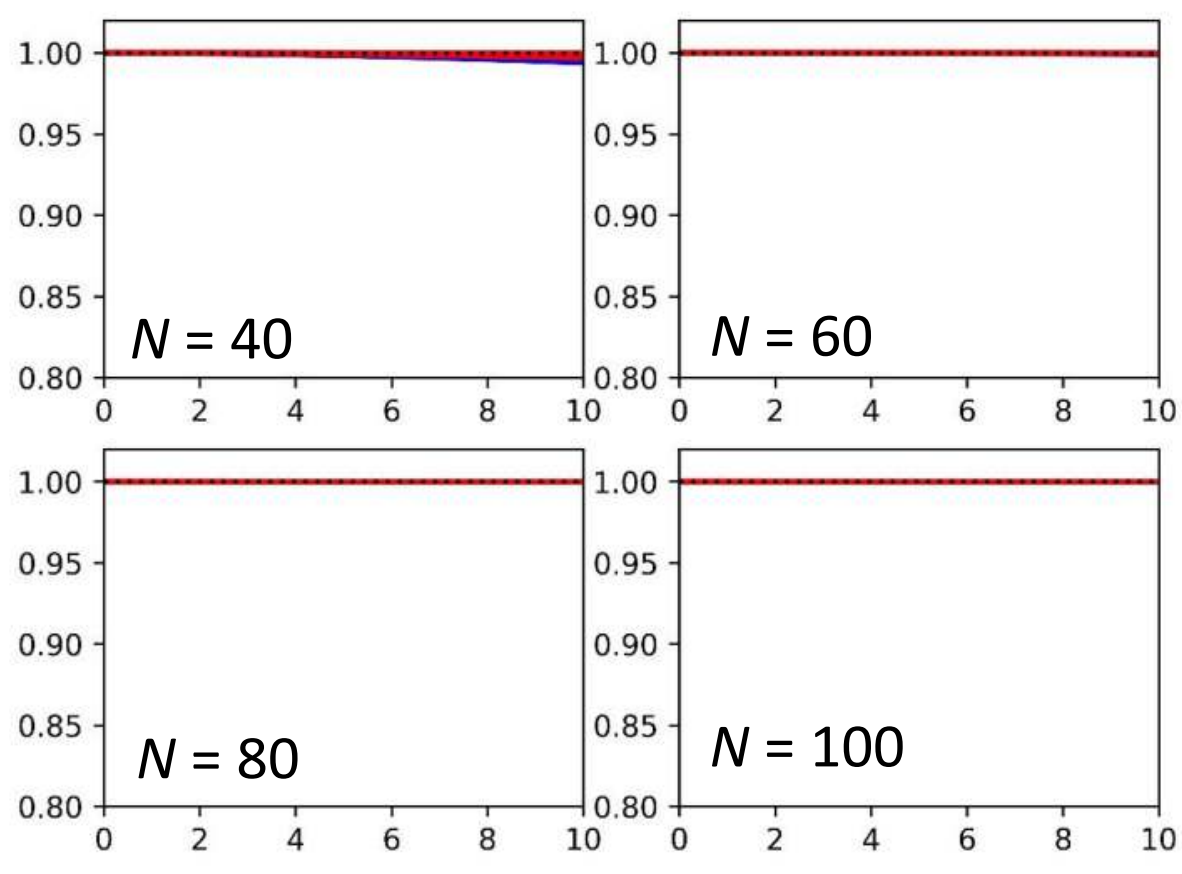

Time $t$ $\left\langle S^{2}\right\rangle_{\text {Sim }}-\left\langle S^{2}\right\rangle_{\text {Exact }}$
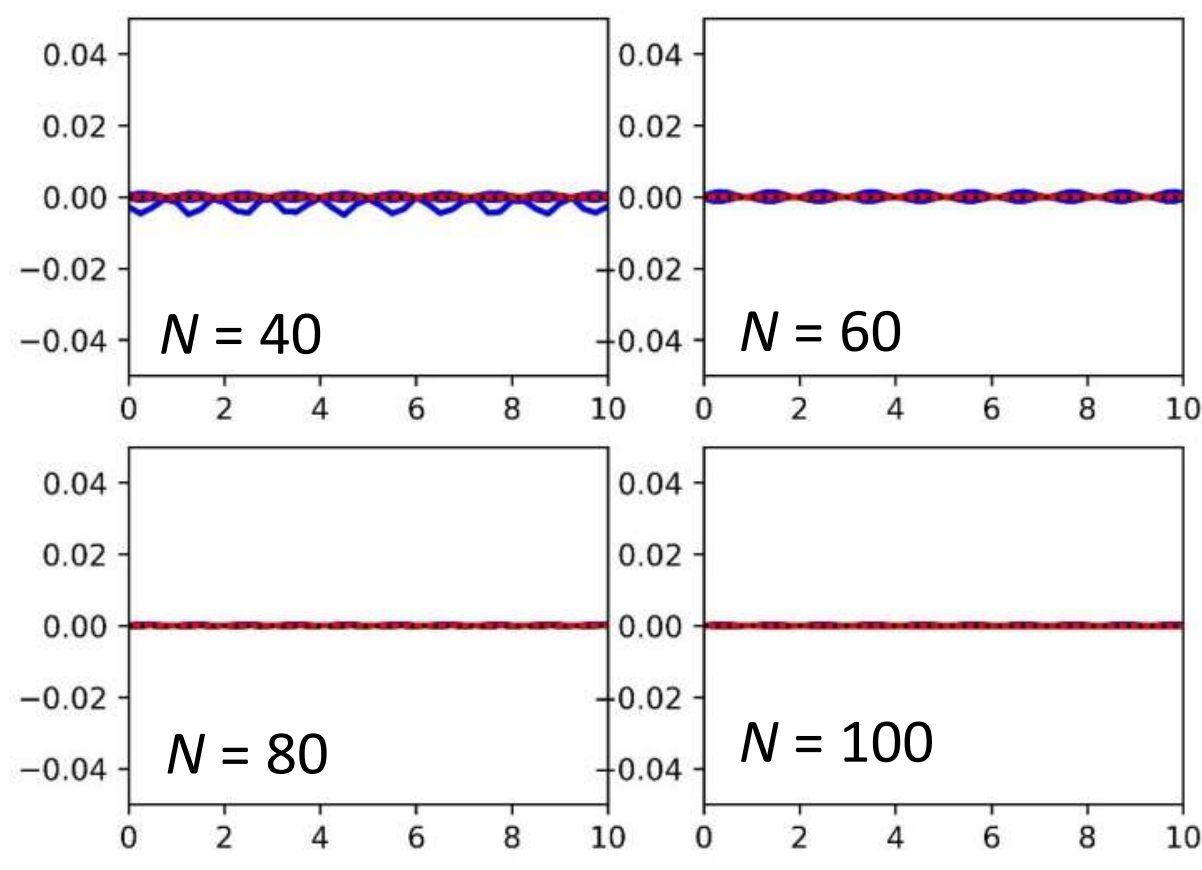

Time $t$ 


\section{QPE for Spin State Discriminations}

(1) Global phase $\rightarrow$ relative phase

(2) Fourier transformation (Time domain $\rightarrow$ Frequency domain)

(3) Measure the first qubit

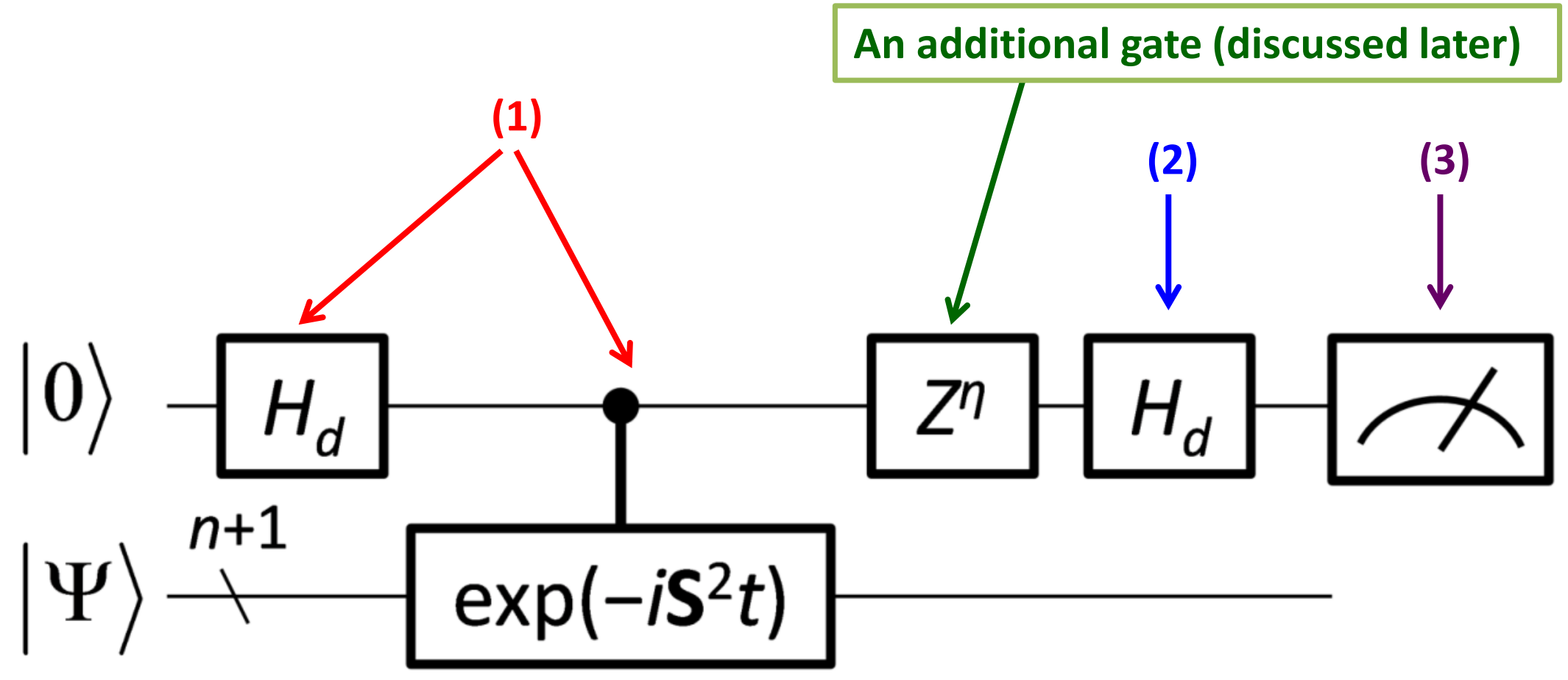




\section{QPE Simulation Results: Two-Spin Systems}

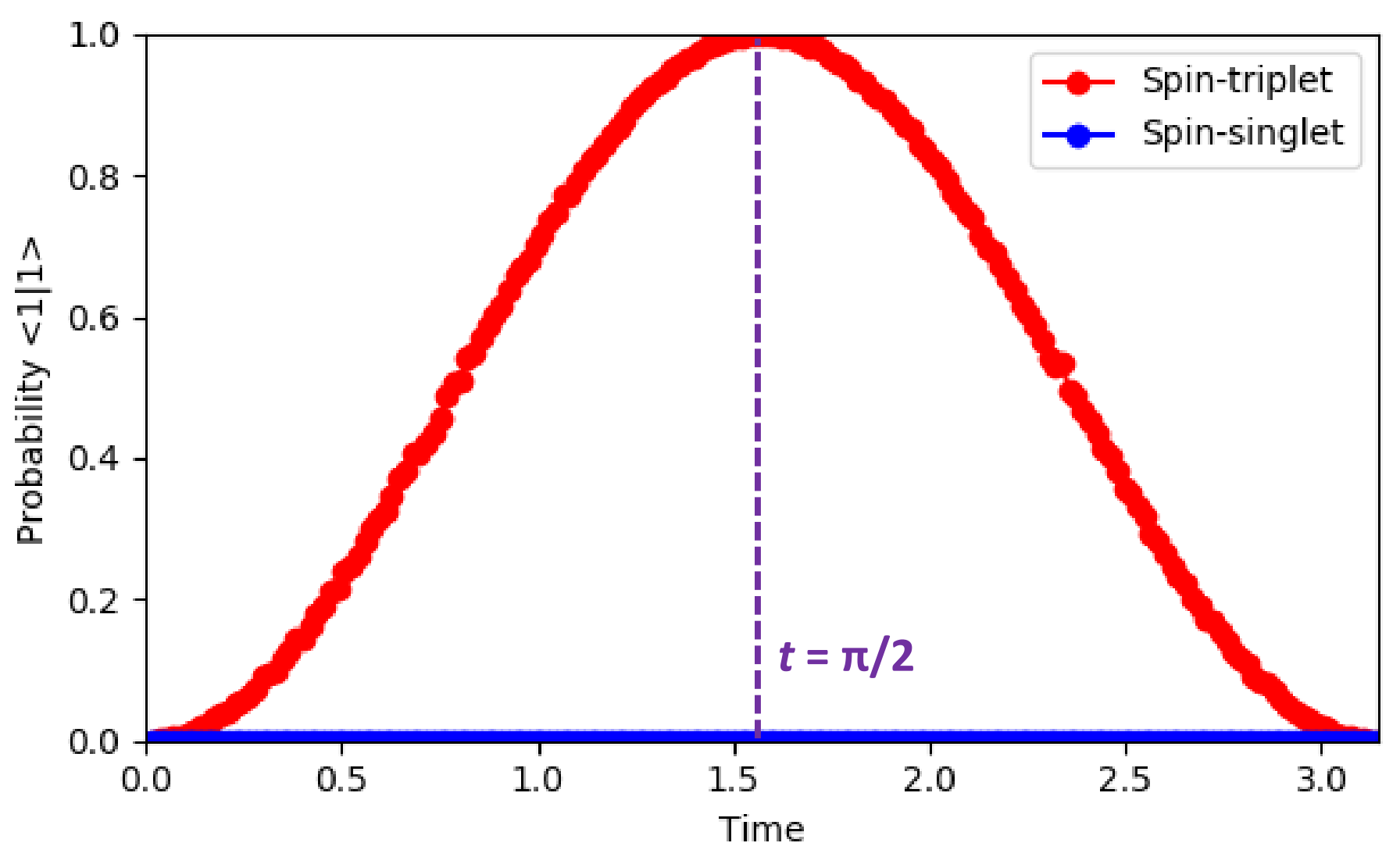

$\exp \left(-i S^{2} t\right)|\Psi(S=1)\rangle=\exp (-2 i t)|\Psi(S=1)\rangle$

$$
\begin{aligned}
& t=\pi / 2 \\
& \exp \left(-i S^{2} t\right)|\Psi(S=1)\rangle=-|\Psi(S=1)\rangle \\
& \exp \left(-i S^{2} t\right)|\Psi(S=0)\rangle=|\Psi(S=0)\rangle
\end{aligned}
$$




\section{QPE Simulation Results: Three-Spin Systems}
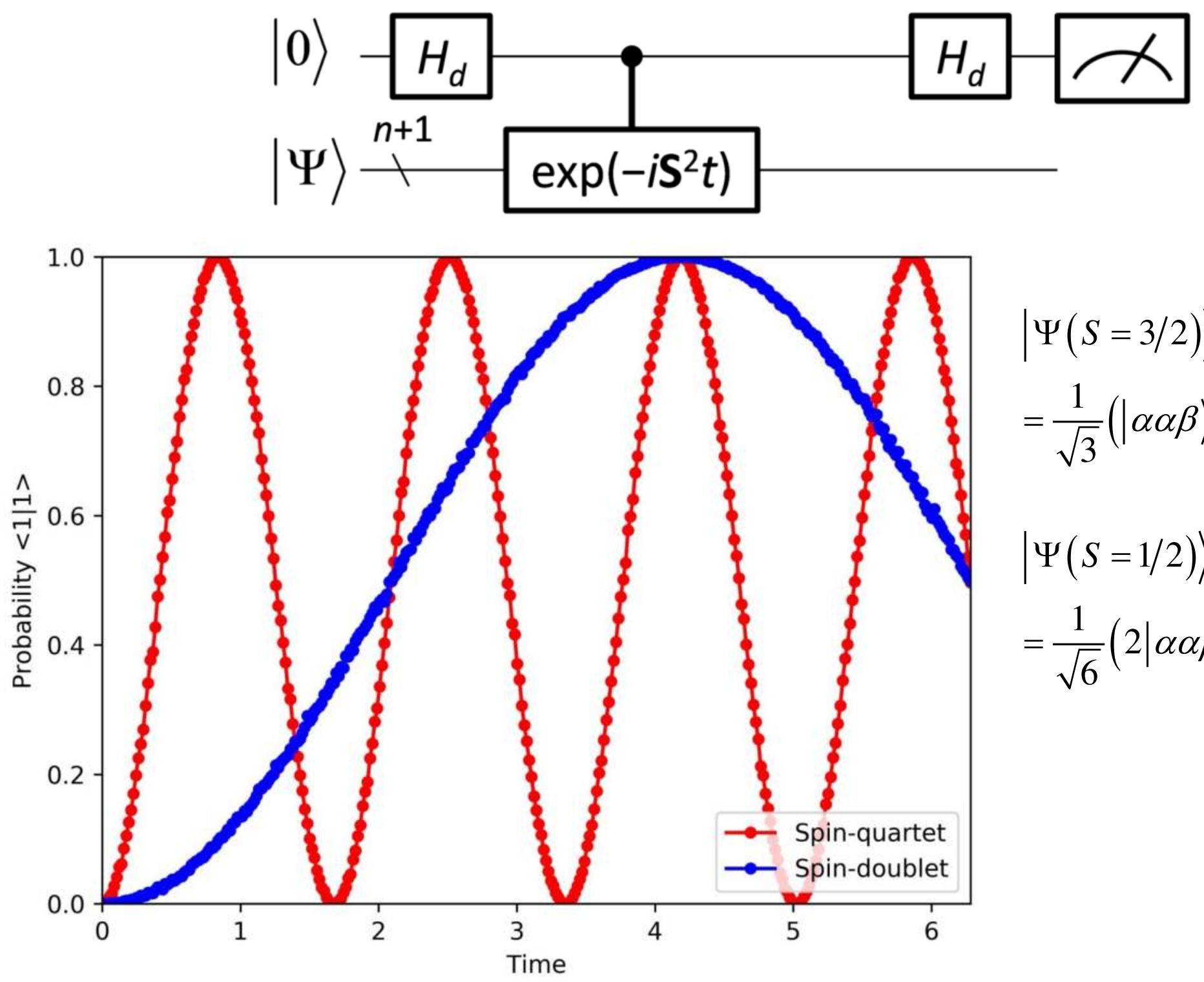

$$
\begin{aligned}
& |\Psi(S=3 / 2)\rangle \\
& =\frac{1}{\sqrt{3}}(|\alpha \alpha \beta\rangle+|\alpha \beta \alpha\rangle+|\beta \alpha \alpha\rangle) \\
& |\Psi(S=1 / 2)\rangle \\
& =\frac{1}{\sqrt{6}}(2|\alpha \alpha \beta\rangle-|\alpha \beta \alpha\rangle-|\beta \alpha \alpha\rangle)
\end{aligned}
$$




\section{QPE Simulation Results: Three-Spin Systems}
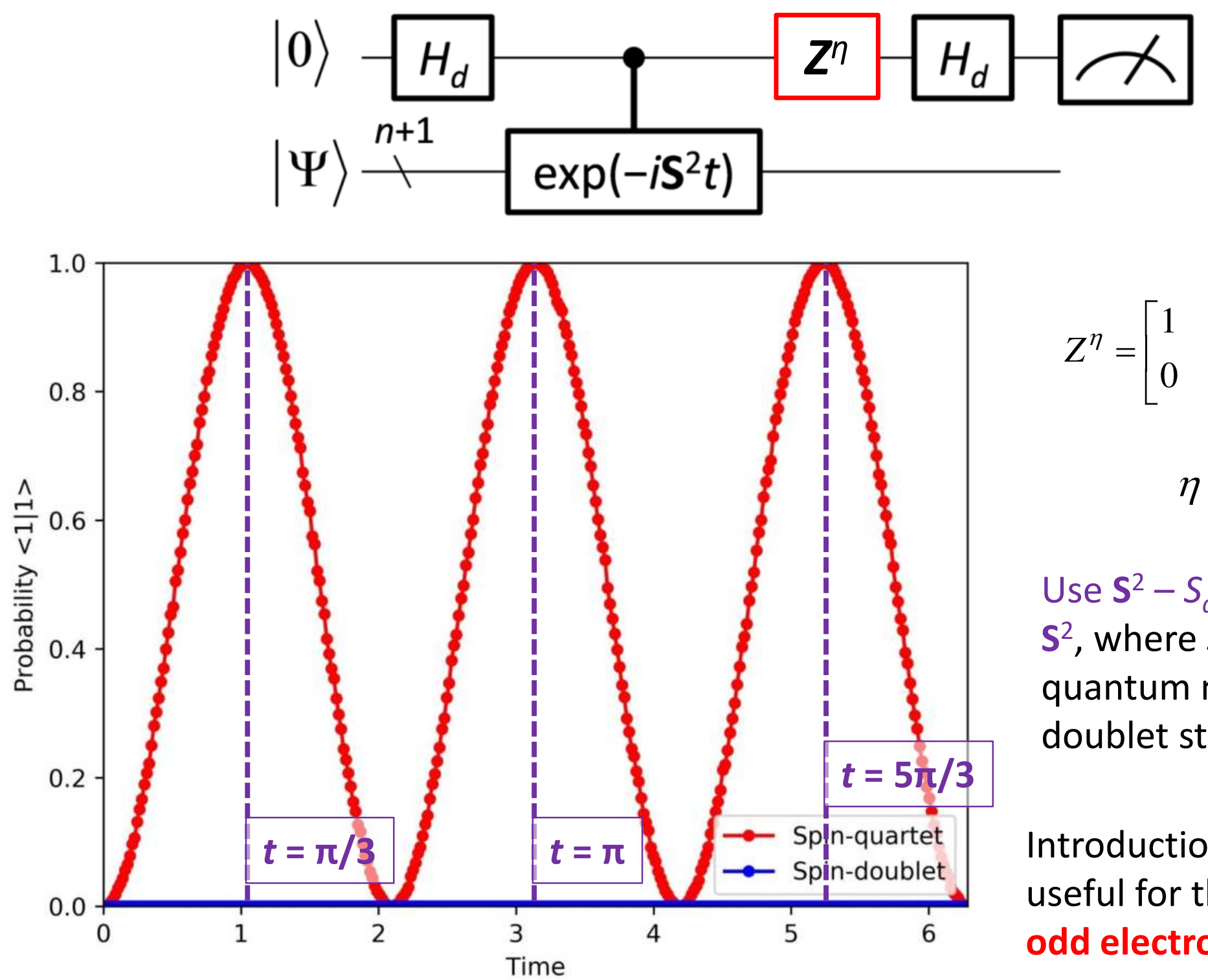

$$
\begin{gathered}
Z^{\eta}=\left[\begin{array}{cc}
1 & 0 \\
0 & \exp (i \eta \pi)
\end{array}\right] \\
\eta=\frac{3}{4 \pi} t
\end{gathered}
$$

Use $S^{2}-S_{d}\left(S_{d}+1\right)$ instead of $S^{2}$, where $S_{d}$ is the spin quantum number of spindoublet state $\left(S_{d}=1 / 2\right)$.

Introduction of $Z^{n}$ is useful for the study of odd electron systems 


\section{Summary}

We proposed a quantum circuit to simulate time evolution of wave functions under the spin operator $\mathbf{S}^{2}$.

By adopting generalized spin coordinate mapping (GSCM), the number of quantum gates required for the simulation is drastically reduced.

We can discriminate spin-singlet and triplet states and spin-doublet and quartet states deterministically, from only one qubit readout in QPE.

Trotter decomposition error is smaller in GSCM than in JWT. 
